\title{
Electron Beam Effects on Silicon Oxide Films - Structure and Electrical Properties
}

Krishna Kanth Neelisetty ${ }^{1,2}$, Sebastian Gutsch ${ }^{3}$, Falk von Seggern ${ }^{2}$, Alan Molinari ${ }^{1,2,}$ Alexander Vahl ${ }^{4}$, Xiaoke $\mathrm{Mu}^{1}$, Torsten Scherer ${ }^{1}$, Chakravadhanula VS Kiran ${ }^{1,5}$ and Christian Kübel ${ }^{1,5,6}$,

1. Institute of Nanotechnology, Karlsruhe Institute of Technology, Eggenstein-Leopoldshafen, Germany

2. TU Darmstadt, Darmstadt, Germany

3. IMTEK Nanotechnologie, Freiburg, Germany

4. Technische Fakultät der CAU Kiel, Institut für Materialwissenschaft, Kiel, Germany

5. Helmholtz Institute Ulm, Karlsruhe Institute of technology, Eggenstein-Leopoldshafen, Germany

6. Karlsruhe Nano- Micro Facility, Karlsruhe Institute of technology, Eggenstein-Leopoldshafen, Germany

As semiconductors, transistors, diodes, and other electronic components are reaching its physical limitations, novel approaches in computation are being pursued. One of them is to mimic the human brain to create artificial neural circuits that have huge applications in the fields of pattern recognition and parallel computing [1] while maintaining ultralow power consumption. One approach to build these networks is an integration of memristive devices where resistance changes are used to realize binary or multinary switches. These advanced electronic components that are an equivalent to synapsis in the human brain, can change their electrical resistance reversibly over a billion cycles. Great efforts have been done to build these devices using a wide range of oxides for real life applications [2]. These devices typically work on the principle of valance change memories where migration of oxygen anions and vacancies result in local redox reactions creating pathways for higher conductivity (low resistive state) and vice versa for lower conductivity (high resistive state).

However, a clear structural insight into the dynamics of these devices is still unclear till date. Very demanding work has been done to bridge this gap using in situ transmission electron microscopy (TEM) to study memristive devices [3]. However, the interpretation and evaluation is complex and sometimes misleading due to the challenging sample preparation using dual beam ion microscopes, the contamination effects [4] and typically effects due to the electron beam [5] during TEM characterization are not addressed. In this paper, we would like to discuss the electron beam induced changes in silicon oxide as a commonly used example system and its influence on the structural changes and hence electrical properties on the nano-electronic devices.

We used $20 \mathrm{~nm}$ silicon oxide TEM support grids (TEM windows SO100-A20Q33A) for imaging. EFTEM investigations are carried out using an aberration (image) corrected FEI titan 80-300 microscope operated at $300 \& 80 \mathrm{kV}$, equipped with Gatan tridium Image filter. EFTEM images are acquired with a $5 \mathrm{eV}$ energy slit centred at an energy loss of $17 \mathrm{eV}$. For in situ electrical measurements, protochips electrical devices (E-AEK11 Finger configuration Pt with $5 \mu \mathrm{m}$ electrode width and $5 \mu \mathrm{m}$ spacing) are used where, silicon oxide is deposited using PE-CVD. A FUSION electrical holder with a Keithley and a Gamry potentiostat were used for electrical characterization.

We have focused on the thin film instability under the influence of electron beam. In Figure 1a-b, an energy filtered TEM (EFTEM) series of a $\mathrm{SiO}_{2}$ thin film imaged at low and high dose is shown. Initially, at a dose of up to $1.9 \mathrm{E} 6 \mathrm{e} / \mathrm{nm}^{2}$ no silicon nanoparticles are visible in the film, only some slight cloudy appearance. At a higher dose, the first particles become visible and the average size of these particles is 
increasing with dose. Moreover, distance between the particles is reduced considerably forming a continuous (connected) network of particles after a dose of $\sim 10 \mathrm{E} 6 \mathrm{e} / \mathrm{nm}^{2}$. This damage occurs both at 80 and $300 \mathrm{kV}$.

The $I / V$ curves in Figure 2 show the effect of the structural changes on the conductivity of the silicon oxide film. Initially, the film as an insulator, but after electron beam exposure with a total dose of $29 \mathrm{E} 6 \mathrm{e} / \mathrm{nm}^{2}$, the $I / V$ curve shows a tremendous increase of the conductivity revealing a typical semiconductor behavior. We assume that this change in conductivity is related to the formation of a continuous silicon-rich network within the oxide film.

\section{References:}

[1] Julian Strobel, et al, 245307 (2017), p. 1.

[2] F. Zahari, et al, AIMS Mater. Sci. 2 (2015), p. 203.

[3] J. Strobel, et al, Appl. Microsc. 46 (2016), p. 206.

[4] M. Hammad Fawey, et al, Microsc. Res. Tech. 79 (2016), p. 615.

[5] S. Gutsch, et al, Beilstein J. Nanotechnol. 6 (2015), p. 964.
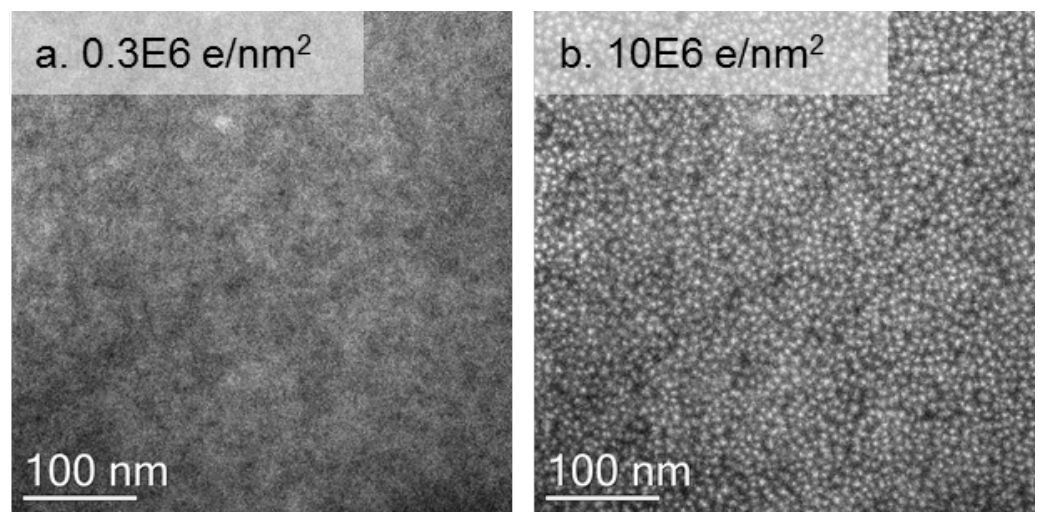

Figure 1. EFTEM micrographs of Si nanoparticle formation. Dose rate: $\sim 5.44 \mathrm{E} 4 \mathrm{e} / \mathrm{nm}^{2} . \mathrm{s}$ a. Dose $0.3 \mathrm{E} 6$ e/nm²; b. 10E6 e/nm².

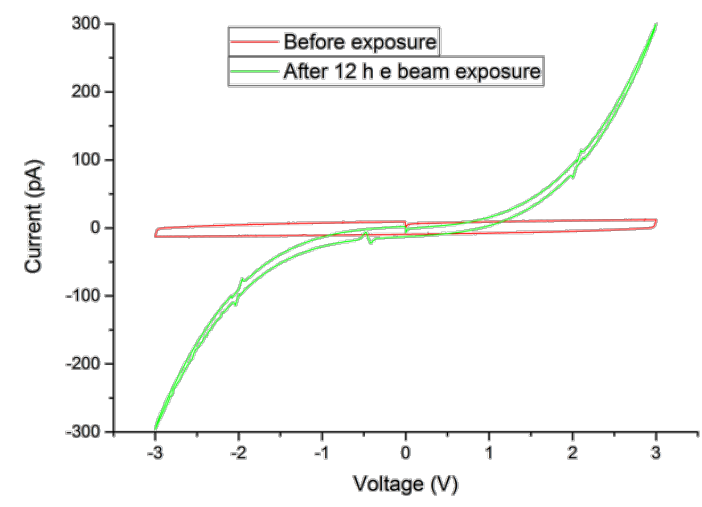

Figure 2. Electrical conductivity $(I V)$ measurement of $\mathrm{SiO}_{2}$ thin film before and after exposure by the electron beam. The IV curves are recorded with the beam off. Dose rate $\sim 500 \mathrm{e} / \mathrm{nm}^{2} . \mathrm{s}$; Total dose $\sim 29 \mathrm{E} 6 \mathrm{e} / \mathrm{nm}^{2}$. 